\title{
Duhuo Jisheng Decoction promotes chondrocyte proliferation through accelerated G1/S transition in osteoarthritis
}

\author{
GUANGWEN WU ${ }^{1}$, WENLIE CHEN ${ }^{1}$, HUAILING FAN $^{2}$, CHUNSONG ZHENG $^{1}$, JIANFENG CHU ${ }^{1}$, \\ RUHUI LIN ${ }^{3}$, JINXIA YE ${ }^{3}$, HUIFENG XU ${ }^{1}$, XIHAI LI ${ }^{1}$, YUNMEI HUANG ${ }^{3}$, \\ HONGZHI YE ${ }^{1}$, XIANXIANG LIU ${ }^{1}$ and MINGXIA WU ${ }^{4}$

\begin{abstract}
Academies of ${ }^{1}$ Integrative Medicine, ${ }^{2}$ Traditional Chinese Medicine and ${ }^{3}$ Integrative Medicine
Biomedical Research Center, Fujian University of Traditional Chinese Medicine, Fuzhou, Fujian 350122;

${ }^{4}$ Second Affiliated People's Hospital of Fujian University of Traditional Chinese Medicine, Fuzhou, Fujian 350003, P.R. China
\end{abstract}

Received May 16, 2013; Accepted June 17, 2013

DOI: 10.3892/ijmm.2013.1481

\begin{abstract}
Duhuo Jisheng Decoction (DHJSD), a well known traditional Chinese folk medicine, is used for eliminating stagnation, removing blood stasis, promoting blood circulation and alleviating pain; it is commonly used for the treatment of various diseases, including osteoarthritis (OA). However, the molecular mechanisms behind the therapeutic effects of OA remain unclear. In the present study, the effects of DHJSD on the morphology of articular cartilage and the G1/S cell cycle progression in chondrocytes, as well as the underlying mechanisms, were investigated. A total of 27 two-month-old male Sprague Dawley rats were randomly divided into 3 groups: the control group (no papain-induced OA; received an equivalent amount of saline only), the model group (papain-induced OA; received an equivalent amount of saline only) and the DHJSD group [papain-induced OA; received a clinical oral dose of DHJSD $(9.3 \mathrm{~g} / \mathrm{kg} / \mathrm{day})]$. After 8 consecutive weeks of treatment, the morphological changes in articular cartilage were observed under an optical microscope and by transmission electron microscopy (TEM) and the mRNA and protein expression levels of cyclin D1, CDK4, CDK6, retinoblastoma protein $(\mathrm{Rb})$ and p16 were measured by RT-PCR and immunohistochemistry, respectively. Treatment with DHJSD significantly improved the arrangement of collagen fibers in the articular cartilage, as well as its structure and reduced cell degeneration compared with the model group. The mRNA and protein expression levels of cyclin D1, CDK4, CDK6 and Rb in the DHJSD-treated group were significantly increased compared with those in the model group, whereas p16 expression was significantly down-
\end{abstract}

Correspondence to: Dr Mingxia Wu, Second Affiliated People's Hospital of Fujian University of Traditional Chinese Medicine, 13 Hudong Road, Gulou District, Fuzhou 350003, P.R. China E-mail:wumingxiafz@163.com

Key words: Duhuo Jisheng Decoction, chondrocyte, proliferation, cell cycle, osteoarthritis regulated. Taken together, these results indicate that DHJSD treatment promotes chondrocyte proliferation by promoting the G1/S checkpoint transition in the cell cycle and by upregulating the expression of cyclin D1, CDK4, CDK6 and Rb and downregulating the expression of p16 and this may, in part, explain its clinical efficacy in the treatment of osteoarthritis.

\section{Introduction}

Osteoarthritis (OA), the most common age-related cartilage and joint disorder (1), is a slowly progressive degenerative disease characterized by the degradation of the extracellular matrix (ECM) and cell death, resulting in a gradual loss of articular cartilage integrity $(2,3)$. The balance of catabolism and anabolism within chondrocytes helps to maintain the structural and functional integrity of the ECM. During the early stages of OA development, cartilage tissues show self-repair activity, the volume of chondrocytes increases and proteoglycan synthesis is accelerated. During the late stages of $\mathrm{OA}$, this balance is broken and the damaging effects of inflammation become more evident. Chondrocytes rapidly respond to changes in the microenvironment of the joint and regulate the dynamic equilibrium between the degradation and synthesis of the ECM, which plays a crucial role in the maintenance of cartilage function (4-7). In a word, the functional changes in chondrocytes play an important role in the degeneration of joint cartilage and chondrocyte proliferation is one of the important factors contributing to the maintenance of cellular function.

The cell cycle plays an important role in the effects on chondrocyte function, which takes place in chondrocytes, leading to division and duplication. Cell cycle control is a highly regulated process that involves a complex cascade of events, in which regulation can be realized through a complex network of cyclins, cyclin-dependent kinases (CDKs) and cyclin-dependent kinase inhibitors (CDKIs). The G1/S checkpoint that exists at the end of the G1 stage is the key point of intracellular and extracellular signaling which integrates into the nucleus, then stimulates $\mathrm{S}$ phase cells to begin a new round of proliferation, differentiation and death, or into the G0 phase $(8,9)$. Briefly, extracellular signals induce the expression of cyclin D1 in cells entering the cell cycle and this binds to and activates CDK4 
and CDK6 (10-12). The ensuing complexes in turn lead to the phosphorylation of retinoblastoma protein $(\mathrm{Rb})$, resulting in its dissociation from the transcription factors, predominantly members of the $\mathrm{E}_{2} \mathrm{~F}$ family, which then activate the many genes required for the progression of the cell cycle to the $S$ phase (10). P16, also known as p16 ${ }^{\mathrm{INK} 4 \mathrm{a}}$, a member of the INK4 family of CDKIs, inhibits CDK4 and CDK6, maintaining Rb in its unphosphorylated $\mathrm{E}_{2} \mathrm{~F}$-associated state and thereby preventing the progression from the G1 to the S phase (13-14). In conclusion, the G1/S progression is highly regulated by cyclin D1, CDK4, CDK6, Rb and p16.

Chinese herbal medicine, a major modality in Traditional Chinese Medicine (TCM) and practiced for thousands of years in China and other Asian countries, is used for the treatment of arthritis and related disorders or 'Bi syndrome' $(15,16)$. Herbal formulas are the common form of administration in Chinese herbal practice and herbal formulas have been well documented in ancient and modern literature (17). According to Chinese herbal theory, interactions among the different herbs in a formula exert a synergistic effect and neutralize the potential toxic and side-effects of the individual constituents $(15,18)$. However, there is as yet a lack of rigorous scientific evaluation of such formulas.

The classical formula, Duhuo Jisheng Decoction (DHJSD), first documented by Sun (19), consists of 15 component herbs, Du Huo (Angelica pubescens), Fang Feng (Saposhnikovia divaricata), Chuan Xiong (Ligusticum chuanxiong), Niu Xi (Achyranthes bidentata), Shang Ji Sheng (Loranthus parasiticus), Qin Jiao (Gentiana macrophylla), Du Zhong (Eucommia ulmoides), Dang Gui (Angelica sinensis), Fu Ling (Poria cocos), Dang Shen (Codonopsis pilosula), Shu Di Huang (Radix rehmanniae preparata), Bai Shao (Radix paeoniae alba), $\mathrm{Xi}$ Xin (Asarum sieboldii), Gan Cao (Glycyrrhiza uralensis) and Rou Gui (Cinnamomum cassia). As a well known traditional Chinese folk medicine, it is used for eliminating wind and dampness, relieving pain due to Bi syndrome, tonifying the liver and kidneys and tonifying the qi and blood; it is commonly used for the treatment of various diseases, including OA. Previous studies have proven that DHJSD has drug- and lead-like compounds with potential synergy and polypharmacology against OA (20). However, to our knowledge, the effects of DHJSD on the proliferation of chondrocytes have not yet been reported. To further elucidate the precise mechanisms behind the therapeutic effects of DHJSD in OA, in the present study, we investigated the effects of DHJSD on the proliferation of chondrocytes. We found that DHJSD promoted chondrocyte G1/S transition, which was accompanied by the upregulation of the expression of cyclin D1, CDK4, Rb and CDK6 and the downregulation of $\mathrm{p} 16$.

\section{Materials and methods}

Materials and reagents. Cyclin D1, CDK4, CDK6, Rb and p16 antibodies were obtained from Santa Cruz Biotechnology, Inc. (Santa Cruz, CA, USA). DNA primers were synthesized by Sangon Biotech (Shanghai, China). The VECTASTAIN ${ }^{\circledR}$ Elite ABC kit (Rabbit IgG) and VECTASTAIN Elite ABC kit (Mouse IgG) were provided by Vector Laboratories, Inc. (Burlingame, CA, USA). The DAB Substrate kit was obtained from Cell Signaling Technology, Inc. (Danvers, MA, USA).
Table I. Composition of Duhuo Jisheng Decoction (DHJSD) formula.

Herb name Relative proportion

Angelica pubescens 3

Saposhnikovia divaricata 2

Ligusticum chuanxiong 2

Achyranthes bidentata 2

Loranthus parasiticus

Gentiana macrophylla

2

Eucommia ulmoides

Angelica sinensis

Poria cocos

Codonopsis pilosula

Radix rehmanniae preparata

2

Radix paeoniae alba

2

Asarum sieboldii

2

Cinnamomum cassia 2

Glycyrrhiza uralensis 2

Papain was obtained from Sigma Chemical Co. (St. Louis, MO, USA).

Herbal preparation. The herbs used in DHJSD, Angelica pubescens, Saposhnikovia divaricata, Ligusticum chuanxiong, Achyranthes bidentata, Loranthus parasiticus, Gentiana macrophylla, Eucommia ulmoides, Angelica sinensis, Poria cocos, Codonopsis pilosula, Radix rehmanniae preparata, Radix paeoniae alba, Asarum sieboldii, Glycyrrhiza uralensis and Cinnamomum cassia, were prepared with traditional methods following harvest (15) and purchased from Tong Ren Tang Pharmaceutical Co. (Beijing, China). The 15 herbs were ground into a powder. DHJSD was formulated by mixing herbal powders in relative proportions according to the Chinese pharmacopoeia (19) (Table I). Stock solutions of DHJSD were prepared by decocting the DHJSD powder in water to a concentration of $3 \mathrm{~g} / \mathrm{ml}$ and stored at $-20^{\circ} \mathrm{C}$.

Animals. Healthy and clean, 27 2-month-old male Sprague Dawley rats were purchased from SLAC Laboratory Animal Co.Ltd. (Shanghai, China) [Laboratory Animal Use Certificate no. SCXK(SH)2007-0005] and raised in a sterile environment. The Experimental Animal Center of Fujian University of TCM offers sterile environment facilities, qualified number SYXK(Min) 2009-0001. All experiments involving the animals complied with the Guidance Suggestions for the Care and Use of Laboratory Animals (2006) administered by the Ministry of Science and Technology of China (21).

Experimental design. Papain-induced rat OA was performed as previously described (22), with slight modifications. Briefly, after 1 week of acclimation, OA was induced in the right knees of 18 rats by an injection of $0.2 \mathrm{ml}$ of $4 \%$ papain solution with $0.1 \mathrm{ml}$ of $0.03 \mathrm{M}$ cystein as an activator on days 1,4 and 7. Two weeks after papain-induced OA, the animals were 
randomly divided into 3 groups: the control group (no papaininduced OA; received an equivalent amount of saline only), the model group (papain-induced OA; received an equivalent amount of saline only) and the DHJSD group [papain-induced OA; received a clinical oral dose of DHJSD (9.3 g/ kg/day)]. All groups were treated once a day for 8 consecutive weeks, following which the animals were sacrificed and tibial plateau cartilage specimens were obtained. The morphological changes were observed under an optical microscope following staining with hematoxylin and eosin (H\&E) and by transmission electron microscopy (TEM). The mRNA and protein levels of cyclin D1, CDK4, CDK6, Rb and p16 were measured by RT-PCR and immunohistochemistry, respectively.

Optical microscopy analyses. The rats were sacrificed after being treated consecutively with or without DHJSD for 8 consecutive weeks. The rat tibial plateau cartilage (inside) specimens, were cut into $5 \times 4 \times 3 \mathrm{~mm}$ sections, fixed in $4 \%$ paraformaldehyde for 2 days, decalcified in $10 \%$ EDTA for 8 weeks, dehydrated in gradient ethanol and subsequently embedded in paraffin. The $4-\mu \mathrm{m}$-thick paraffin sections were cut using a microtome, dewaxed in xylene and stained with $H \& E$. The changes in cartilage morphological were observed under a microscope (Olympus, Tokyo, Japan) and images were acquired at a magnification of $\times 200$.

TEM. The rats were sacrificed after being treated consecutively with or without DHJSD for 8 weeks. The tibial plateau cartilage (outside) specimens, were cut into $1 \times 1 \times 2 \mathrm{~mm}$ sections, pre-fixed in $4 \%$ glutaraldehyde and $1.5 \%$ paraformaldehyde solution ( $\mathrm{pH} \mathrm{7.3)}$ ) at $4^{\circ} \mathrm{C}$ for 3 days, post-fixed with $1 \%$ osmium tetroxide at $4^{\circ} \mathrm{C}$ for $2 \mathrm{~h}$ after being decalcified in 5.5\% EDTA for 12 weeks at $4^{\circ} \mathrm{C}$. The specimens were dehydrated with graded alcohol-acetone and embedded in Epon-618 resin. After the $1-\mu \mathrm{m}$-thick resin semi-thin sections were cut using a microtome and stained with azur-methylene blue, the structure of the cartilage was observed and the ultra-thin sections were observed under an optic microscope. The 70-nm ultrathin sections were cut using a Leica ultramicrotome, stained with $2 \%$ aqueous uranyl acetate and counterstained with $0.3 \%$ lead citrate. The ultrastructure of the articular cartilage was observed under a transmission electron microscope (Hitachi H7650; Shangai, China).

RNA extraction and RT-PCR analysis. Following treatment with or without DHJSD for 8 consecutive weeks, the rats were sacrificed and tibial plateau cartilage specimens were obtained from each group. Total RNA from the tibial plateau cartilage specimens was isolated using TRIzol reagent (Invitrogen Life Technologies, Carlsbad, CA, USA) after being grinded in liquid nitrogen using a mortar. Oligo(dT)-primed RNA $(1 \mu \mathrm{g})$ was reverse-transcribed using SuperScript II reverse transcriptase (Promega, Madison, WI, USA) according to the manufacturer's instructions. The obtained cDNA was used to determine the mRNA levels of cyclin D1, CDK4, CDK6, $\mathrm{Rb}$ and $\mathrm{p} 16$ by PCR suing Taq DNA polymerase (Fermentas/ Thermo Fisher Scientific, Pittsburgh PA, USA). $\beta$-actin was used as an internal control. The primers and the annealing temperature $\left({ }^{\circ} \mathrm{C}\right)$ used for the amplification of cyclin D1, CDK4, CDK6, Rb, p16 and $\beta$-actin transcripts were as follows: cyclin D1 sense, 5'-GAC ACC AAT CTC CTC AAC GAC-3' and antisense, 5'-AGA CAA GAA ACG GTC CAG GTA G-3' (216 bp, 55 $\mathrm{C})$; CDK4 sense, 5'-CCT ACG GAC ATA CCT GGA CAA-3' and antisense, 5'-GAG GCA ATC CAA TGA GAT CAA-3' (404 bp, 55 C); CDK6 sense, 5'-GTT TCA GCT TCT CCG AGG TCT-3' and antisense, 5'-CGT CAA GCA TTT CAG AAG GAG-3' (469 bp, 55 ${ }^{\circ} \mathrm{C}$ ); Rb sense, 5'-CTT TAT TGG CCT GTG CTC TTG-3' and antisense, 5'-ATT CCA TGA TTC GAT GCT CAC-3' (225 bp, $\left.55^{\circ} \mathrm{C}\right)$; p16 sense, 5'-GCT CTC CTG CTC TCC TAT GGT-3' and antisense, 5'-AGA AGT TAT GCC TGT CGG TGA-3' (268 bp, 55 ${ }^{\circ} \mathrm{C}$ ); $\beta$-actin sense, 5'-GGG AAG TGC TGG ATA G-3' and antisense, 5'-GTG ATG TTT CGG ATG G-3' (453 bp, $55^{\circ} \mathrm{C}$ ).

Immunohistochemical analysis. Following treatment with or without DHJSD for 8 consecutive weeks, the rats were sacrificed and the rat tibial plateau cartilage (inside) specimens from each group, were cut into $5 \times 4 \times 3 \mathrm{~mm}$ sections, fixed in $4 \%$ paraformaldehyde for 2 days, decalcified in 10\% EDTA for 8 weeks, dehydrated in gradient ethanol and embedded in paraffin. The $5-\mu$ m-thick paraffin sections were cut using a microtome, dewaxed in xylene and analyzed by immunohistochemistry. The sections were then incubated with sodium citrate buffer (10 mM sodium citrate, $\mathrm{pH}$ 6.0) in a microwave for antigen retrieval. Endogenous peroxidase activity of the sections was quenched by incubating in PBS containing $0.3 \% \mathrm{H}_{2} \mathrm{O}_{2}$ and $0.3 \%$ Triton $\mathrm{X}-100$ for $30 \mathrm{~min}$ following repeated washing in PBS. Immunohistochemical staining was performed using the VECTASTAIN elite ABC kit according to the manufacturer's instructions. Briefly, after blocking with normal serum in PBS, the sections were treated with an optimal dilution of primary antibody overnight at $4^{\circ} \mathrm{C}$ (cyclin D1, 1:100; CDK4, 1:150; CDK6, 1:100; Rb, 1:80 and p16, 1:100). The sections were incubated with a biotinylated anti-rabbit or mouse $\operatorname{IgG}$ antibody for $60 \mathrm{~min}$ and then treated with ABC reagent for $60 \mathrm{~min}$. They were finally treated with DAB for $5 \mathrm{~min}$. Subsequently, they were dehydrated with ascending concentrations of ethanol solutions, cleared with xylene and mounted on a coverslip using neutral gum. After staining, 5 random fields (x100) were randomly selected in each slide and the average proportion of positive cells in each field was counted using the true color multi-functional cell image analysis management system. To rule out any non-specific staining, PBS was used to replace the primary antibody as the negative control.

Statistical analysis. Data were analyzed using statistical software SPSS 13.0. All data are presented as the means \pm standard deviation. Statistical analysis of the data was performed using the Student's t-test and one-way analysis of variance (ANOVA). A p-value $<0.05$ was considered to indicate a statistically significant difference.

\section{Results}

Effect of DHJSD on the morphology of articular cartilage. To determine the effects of DHJSD on cartilage morphology, the sections were stained with H\&E and observed under an optical microscope. As shown in Fig. 1A, in the control group, the 4 layer structures (including surface layer, transitional layer, radiation layer and calcification layer) of articular carti- 

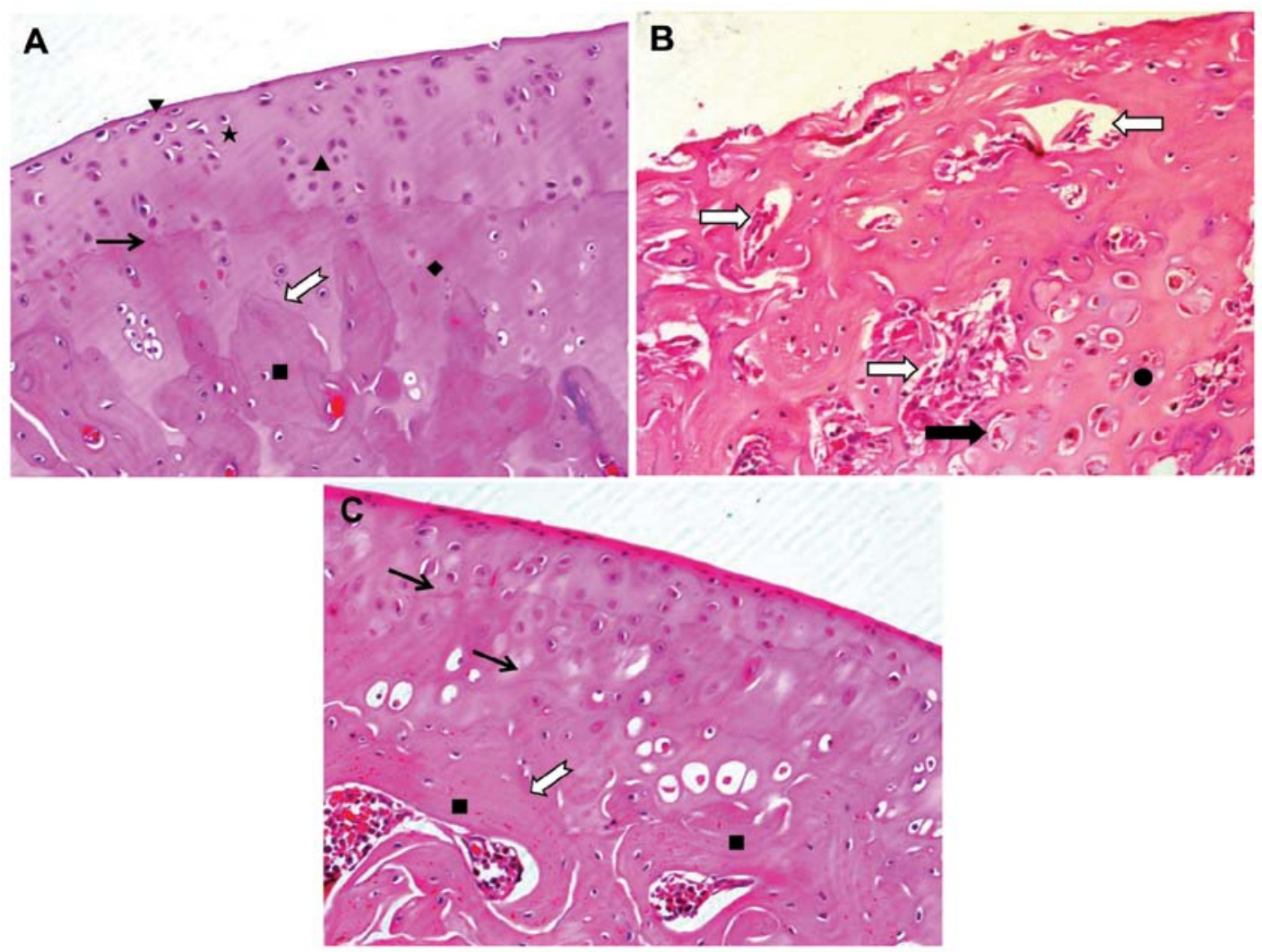

Figure 1. Effect of Duhuo Jisheng Decoction (DHJSD) on the microstructure of cartilage tissue. Following treatment with or without DHJSD for 8 consecutive weeks, the rats were sacrificed and tibial plateau cartilages (inside) specimens from each group were processed for H\&E staining. (A) Control group; (B) model group; (C) DHJSD group. The morphological changes in the cartilage were observed under a microscope and images were aquired at a magnification of $\mathrm{x} 200$. $\mathbf{\nabla}$, Surface layer; $\star$, transitional layer; $\mathbf{\Delta}$, radiation layer; $\bullet$, calcification layer; $\mathbf{m}$, subchondral bone; $\rightarrow$, tidal line; $\Rightarrow$, cement line; $\Rightarrow$, bone marrow vascular invasion into the cartilage layer; $\boldsymbol{-}$, larger cartilage capsule on the calcification layer; $\bullet$, denatured or necrotic chondrocytes on the calcification layer.

lage were visible. The tidal line between the radiation and calcification layer was complete. The cement line between the calcified layer and subchondral bone was obvious, winding. The surface was smooth and the collagen fibers formed a complete perichondrium. The chondrocytes appeared oblate in the surface layer and oval in the transitional and radiation layer and were arranged regularly, with a blue nucleus and red cytoplasm. The cartilage matrix was purple red. However, in the model group, the articular cartilage structure was disordered. The tidal line, as well as the cement line between the calcified layer and subchondral bone had disappeared and bone marrow vascular invasion into the cartilage layer was observed. The surface layer was damaged and the complete perichondrium had disappeared, leaving a velvet-like structure. The number of chondrocytes on the surface and deep layer was significantly reduced, as many cells appeared necrotic. The cartilage capsule became large and many chondrocytes appeared denatured or necrotic on the calcification layer. The cartilage matrix appeared red with an increase in collagen fiber content (Fig. 1B). However, following treatment with DHJSD, the structure of the articular cartilage significantly improved; 2 tidal lines, as well as the cement line between the calcified layer and subchondral bone were obvious, winding and no bone marrow vascular invasion into the cartilage layer was observed. The surface was much smoother and the collagen fibers formed a complete perichondrium, compared with the model group. The total number of chondrocytes and regularly arranged chondrocytes were significantly increased and cell degeneration was significantly reduced compared with the model group. The cartilage matrix appeared purple red (Fig. 1C). Collectively, these findings suggest that DHJSD is effective in the treatment of OA.

Chondrocytes, the only type of cell present in cartilage, play a central role in the equilibrium between the anabolism and catabolism of the fundamental component of cartilage. In order to further clarify the mechanisms through which DHJSD exerts its therapeutic effects in OA, we observed the ultrastructural changes in the chondrocytes by TEM. As shown in Fig. 2A and B, in the control group, the chondrocytes appeared to have an almost oval shape or a triangular shape with many microvilli-like protrusions. The cells contained more organelles, such as an abundant rough endoplasmic reticulum, a mature Golgi apparatus, some mitochondria and glycogen particles scattered in the cytoplasm. In the model group, many chondrocytes appeared denatured and necrotic and the organelles were destroyed or had disappeared (Fig. 2C and D). Following treatment with DHJSD for 2 months, the chondrocytes contained more organelles and the rough endoplasmic reticulum was abundant and the Golgi apparatus was much more mature, suggesting that protein synthesis and processing had increased; the mitochondria were abundant, suggesting an enhanced cellular energy (Fig. 2E and F). More chondrocytes 

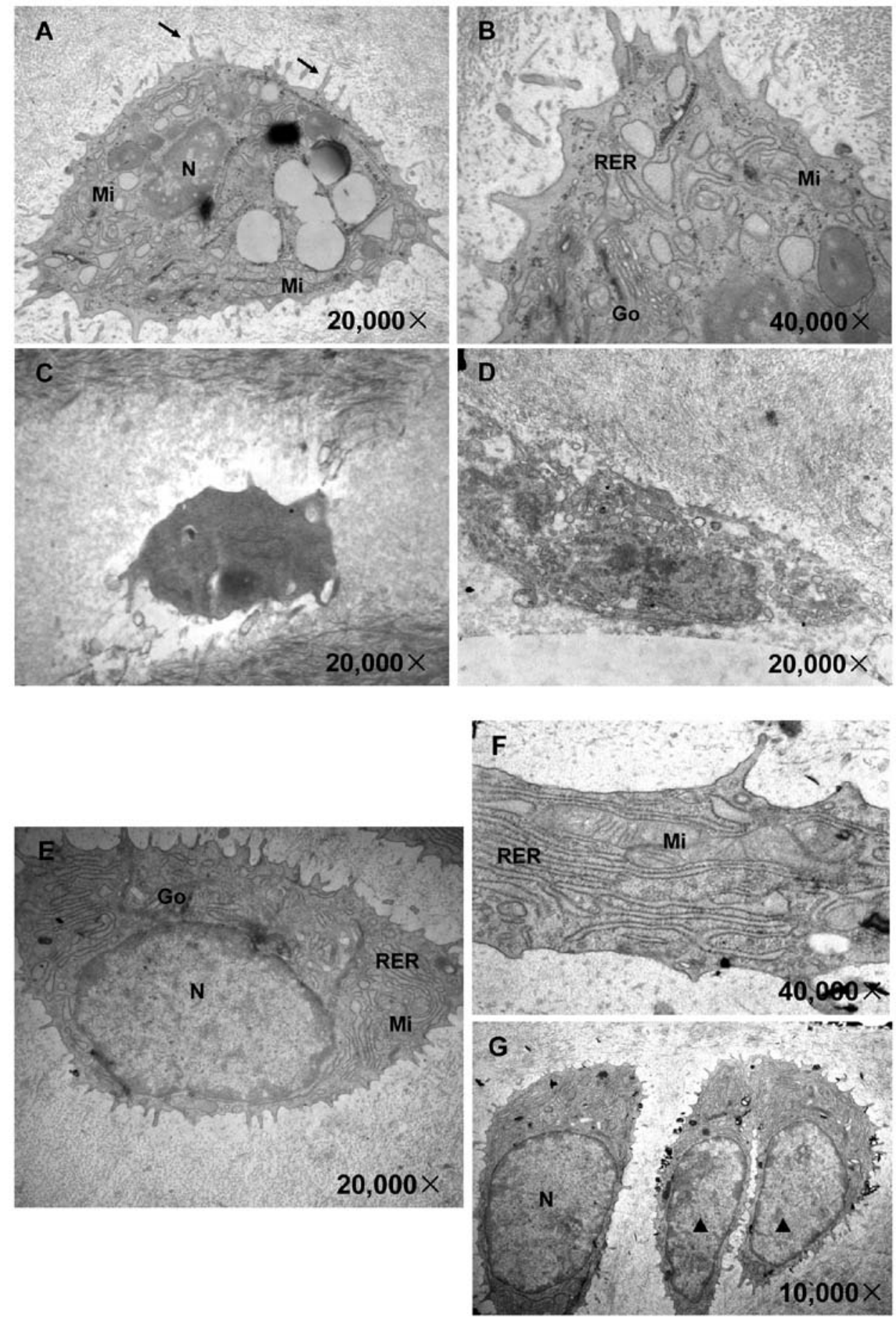

Figure 2. Effect of Duhuo Jisheng Decoction (DHJSD) treatment on the ultrastructure of chondrocytes. Following treatment with or without DHJSD for 8 consecutive weeks, the rats were sacrificed and the ultrastructure of the tibial plateau cartilage (outside) was observed under a transmission electronic microscope. The images were acquired at a magnification of $\mathrm{x} 10,000, \mathrm{x} 20,000$ or $\mathrm{x} 40,000$ magnification. (A and B) Control group; (C and D) model group; (E, F and G) DHJSD group. N, nuclei; $\rightarrow$, microvilli-like protrusions; RER, rough endoplasmic reticulum; Go, Golgi apparatus; Mi, mitochondria; $\mathbf{\Lambda}$, homologous chondrocytes.

were observed, with many near triangular homologous cells in the cartilage, suggesting that DHJSD promoted chondrocyte proliferation (Fig. 2G).

Effect of DHJSD on the expression of cyclin D1, CDK4, $C D K 6, R b$ and p16. It is well known that the G1/S transition is dependent on the activity of cyclin D1-CDK4/6. Once activated, these CDK complexes phosphorylate $\mathrm{Rb}$, resulting in its dissociation from the transcription factors, predominantly members of the $\mathrm{E}_{2} \mathrm{~F}$ family, which then activate the many genes required for the progression of the cell cycle to the $\mathrm{S}$ phase. This progression is controlled by p16, which causes transient or permanent cell cycle arrest in cells with DNA damage. To further explore the mechanism behind the promotion of chondrocyte proliferation by DHJSD, we analyzed the mRNA and protein expression levels of cyclin D1, CDK4, 

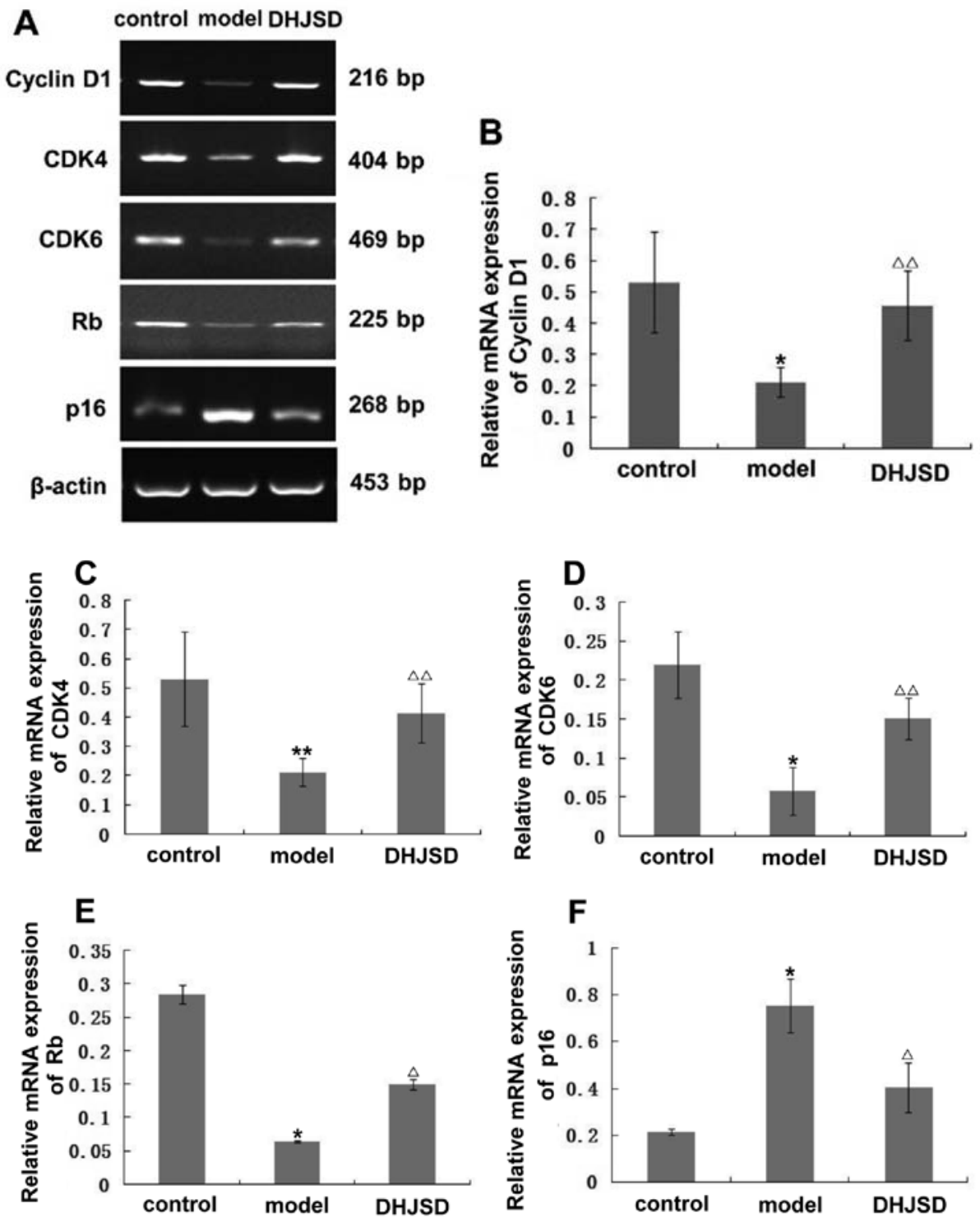

Figure 3. Effect of Duhuo Jisheng Decoction (DHJSD) on the mRNA expression of cyclin D1, CDK4, CDK6, Rb and p16. Following treatment with or without DHJSD for 8 consecutive weeks, the rats were sacrificed and tibial plateau cartilage specimens from each group were obtained. The mRNA levels of cyclin D1, $\mathrm{CDK} 4, \mathrm{CDK} 6, \mathrm{Rb}$ and $\mathrm{p} 16$ in the chondrocytes were determined by RT-PCR. $\beta$-actin was used as an internal control. For each group, RT-PCR analyses were performed in triplicate, as the cartilage from 3 rats was randomly mixed together once. Data shown are the means \pm SD (error bars). (A) The representative amplified products of cyclin D1, CDK4, CDK6, Rb and p16. The relative mRNA expression of (B) cyclin D1, (C) CDK4, (D) CDK6, (E) Rb and (F) p16. "p<0.01, ${ }^{* *} \mathrm{p}<0.05$, statistically significant versus control group. ${ }^{\wedge} \mathrm{p}<0.01,{ }^{\Delta \wedge} \mathrm{p}<0.05$, statistically significant versus model group.

CDK6, Rb and p16 following treatment with DHJSD using RT-PCR and immunohistochemistry, respectively. As shown in Fig. 3A, the amplified products of cyclin D1, CDK4, CDK6, Rb and p16 were clearly visible on the agarose gel. Quantification of the PCR products indicated that the levels of cyclin D1, CDK4, CDK6, Rb were significantly lower, but those of p16 were higher in the model group compared with the control group $(p<0.01$ or 0.05$)$. However, following treatment with DHJSD, the levels of cyclin D1, CDK4, CDK6, Rb increased and those of p16 decreased, significantly versus the model group $(\mathrm{p}<0.01$ or 0.05$)$; the protein expression pattern of cyclin D1, CDK4, CDK6, Rb and p16 was similar to their respective mRNA levels (Figs. 4-8). These results suggest that DHJSD promotes chondrocyte proliferation by upregulating the expression of cyclin D1, CDK4, CDK6 and Rb and downregulating the expression of p16.

\section{Discussion}

OA is caused by multiple molecular abnormalities, rather than being the result of a single effect (23). In this regard, the application of combinational drugs or multi-target drugs, in which more than 2 drugs interact with multiple targets simultaneously, may be a rational and effective treatment strategy for OA (20). Notably, TCM is a holistic approach to health that attempts to bring the body, mind and spirit into harmony; thus, it advocates drug-combined administrations (24). Furthermore, previous studies have demonstrated that Chinese medicinal herbs have the potential to ameliorate the progression of OA and such herbs have received increasing attention $(25,26)$. DHJSD is a classical formula, which is commonly used for the treatment OA. Our study demonstrates that treatment with DHJSD promotes the cell cycle G1/S transition in chondro- 

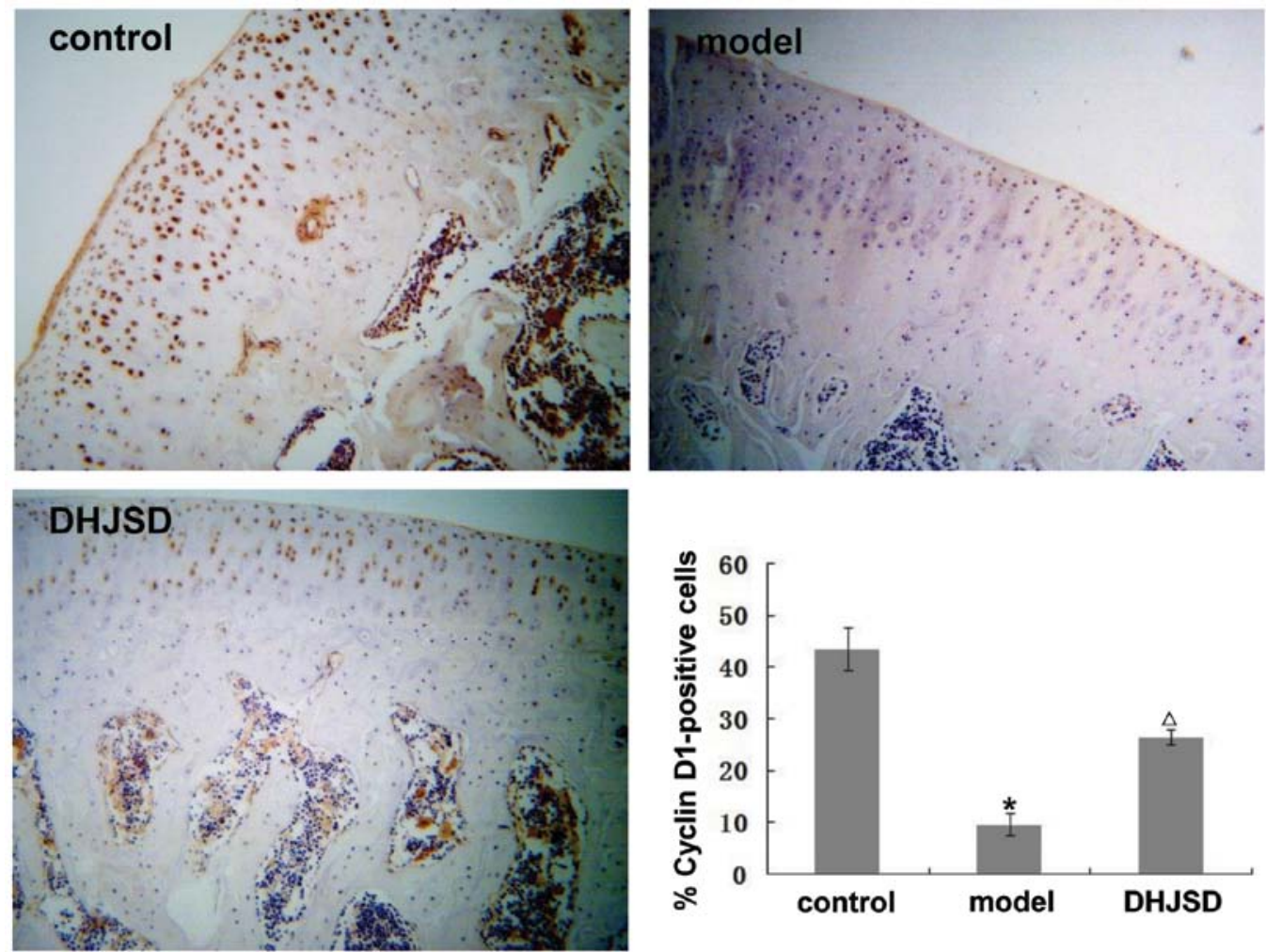

Figure 4. Effect of Duhuo Jisheng Decoction (DHJSD) on the protein expression of cyclin D1. Following treatment with or without DHJSD for 8 consecutive weeks, the rats were sacrificed and tibial plateau cartilage (inside) speciments from each group were processed for immunohistochemical staining of cyclin D1 The images were acquired at a magnification of x100. Results from the quantification of immunohistochemical assay are represented as a percentage of positively-stained cells. Data shown are the means \pm SD (error bars) from 9 individual rats in each group. ${ }^{*} \mathrm{p}<0.01$, statistically significant versus control group. ${ }^{\wedge} \mathrm{p}<0.01$, statistically significant versus model group
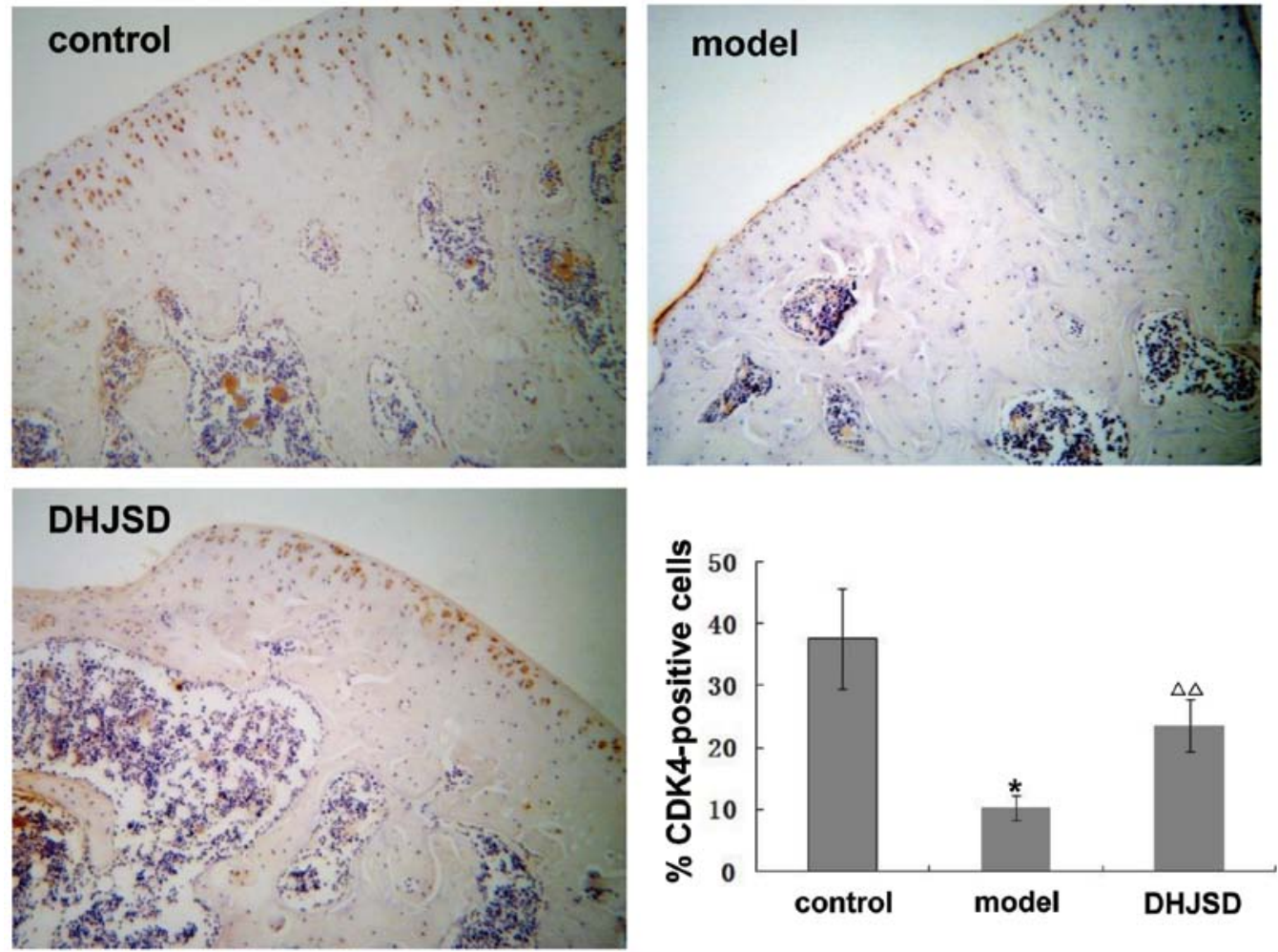

Figure 5. Effect of Duhuo Jisheng Decoction (DHJSD) on the protein expression of CDK4. Following treatment with or without DHJSD for 8 consecutive weeks, the rats were sacrificed and tibial plateau cartilage (inside) specimens from each group were processed for immunohistochemical staining of CDK4. The images were acquired at a magnification of $\mathrm{x} 100$. Results from the quantification of immunohistochemical assay are represented as the percentage of positively-stained cells. Data shown are the means \pm SD (error bars) from 9 individual rats in each group. ${ }^{*} \mathrm{p}<0.01$, statistically significant versus control group. ${ }^{\Delta \Delta} \mathrm{p}<0.05$, statistically significant versus model group. 

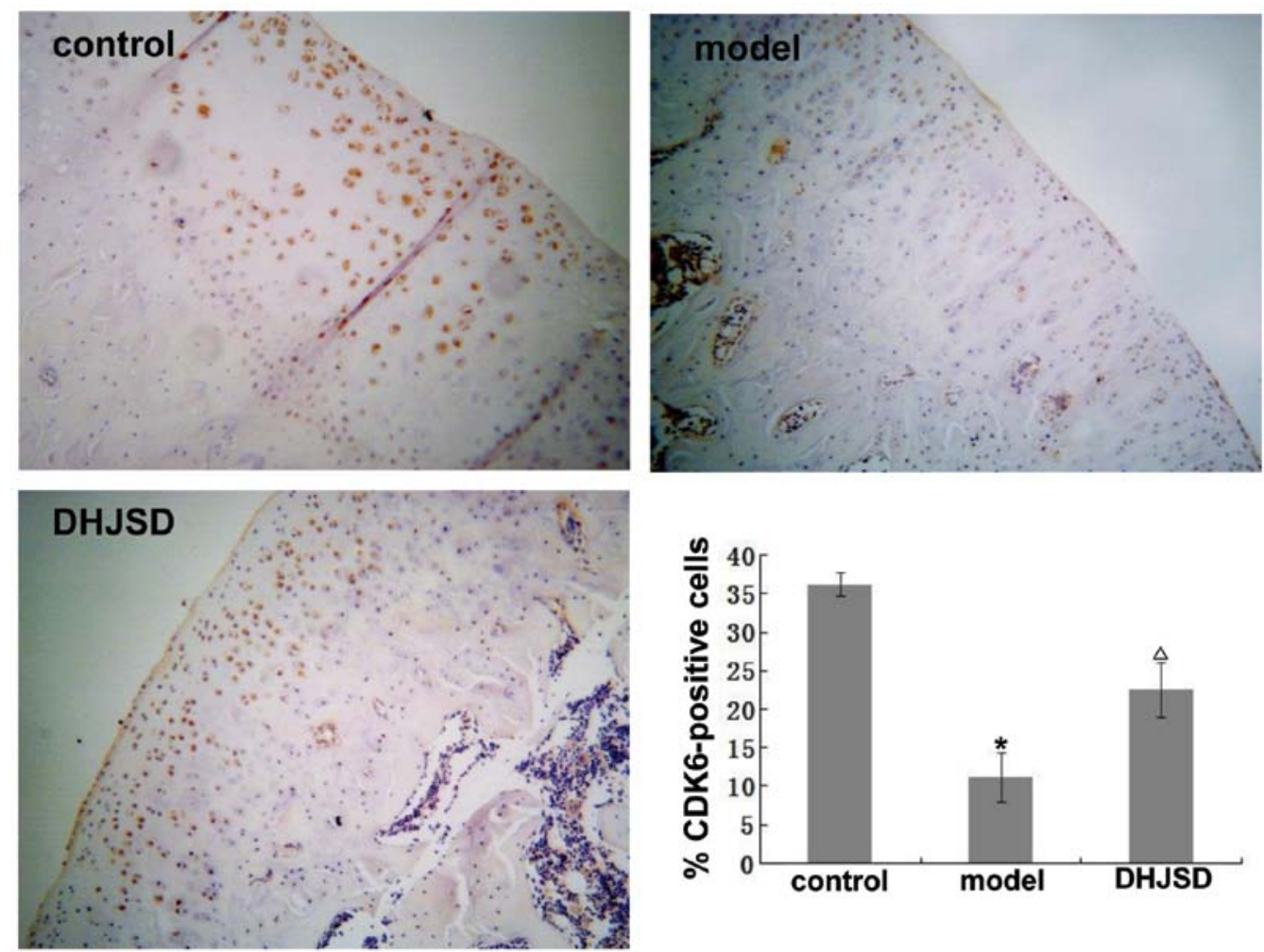

Figure 6. Effect of Duhuo Jisheng Decoction (DHJSD) on the protein expression of CDK6. Following treatment with or without DHJSD for 8 consecutive weeks, the rats were sacrificed and tibial plateau cartilage (inside) specimens from each group were processed for immunohistochemical staining of CDK6. The images were acquired at a magnification of $\mathrm{x} 100$. Results from the quantification of immunohistochemical assay are represented as the percentage of positively-stained cells. Data shown are the means \pm SD (error bars) from 9 individual rats in each group. ${ }^{*} p<0.01$, statistically significant versus control group. ${ }^{\Delta} \mathrm{p}<0.01$, statistically significant versus model group.
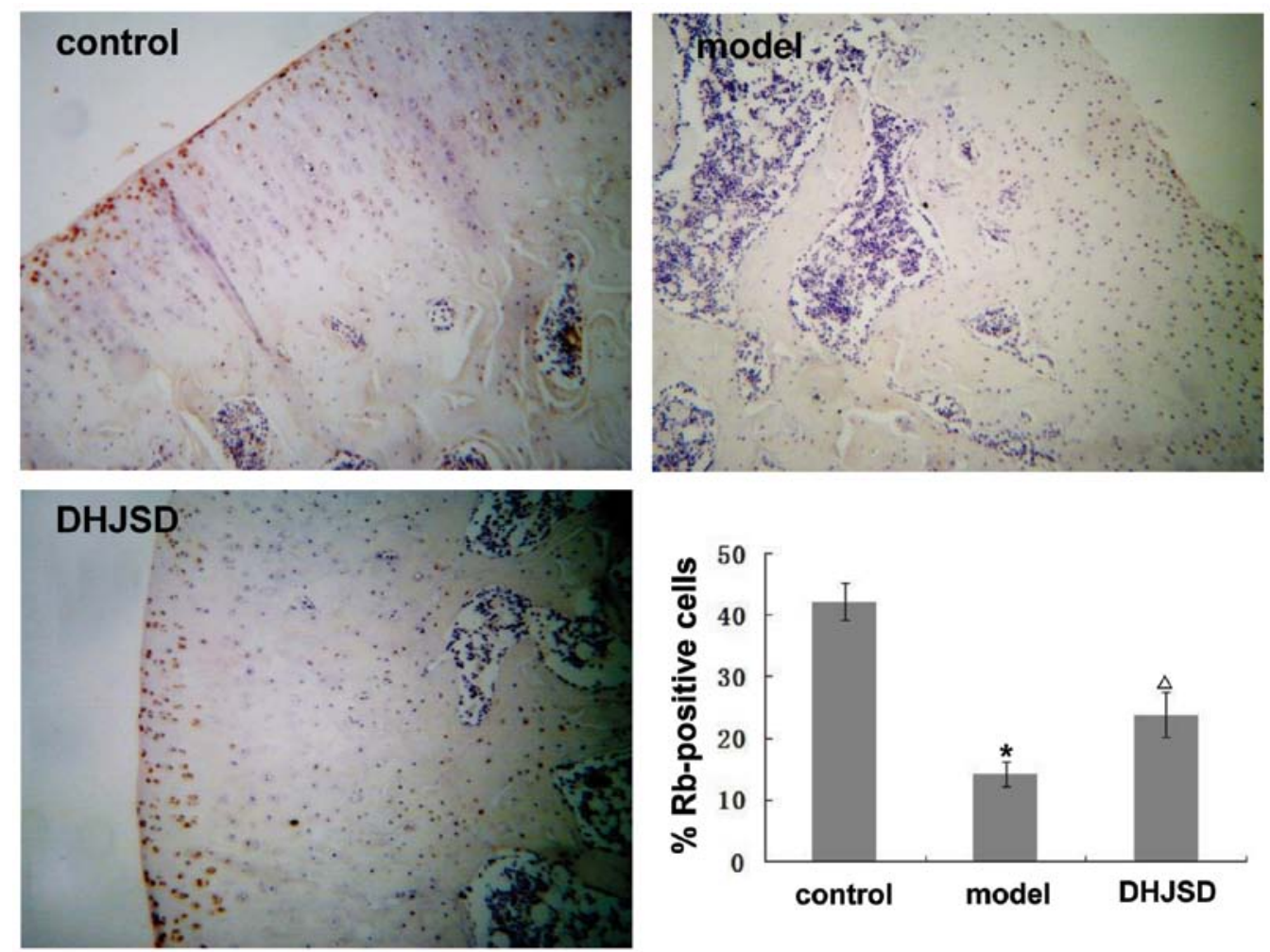

Figure 7. Effect of Duhuo Jisheng Decoction (DHJSD) on the protein expression of Rb. Following treatment with or without DHJSD for 8 consecutive weeks, the rats were sacrificed and tibial plateau cartilage (inside) specimens from each group were processed for immunohistochemical staining of Rb. The images were acquired at a magnification of $x 100$. Results from the quantification of immunohistochemical assay are represented as the percentage of positively-stained cells. Data shown are the means $\pm \mathrm{SD}$ (error bars) from 9 individual rats in each group. ${ }^{*} \mathrm{p}<0.01$, statistically significant versus control group. ${ }^{\Delta} \mathrm{p}<0.01$, statistically significant versus model group. 

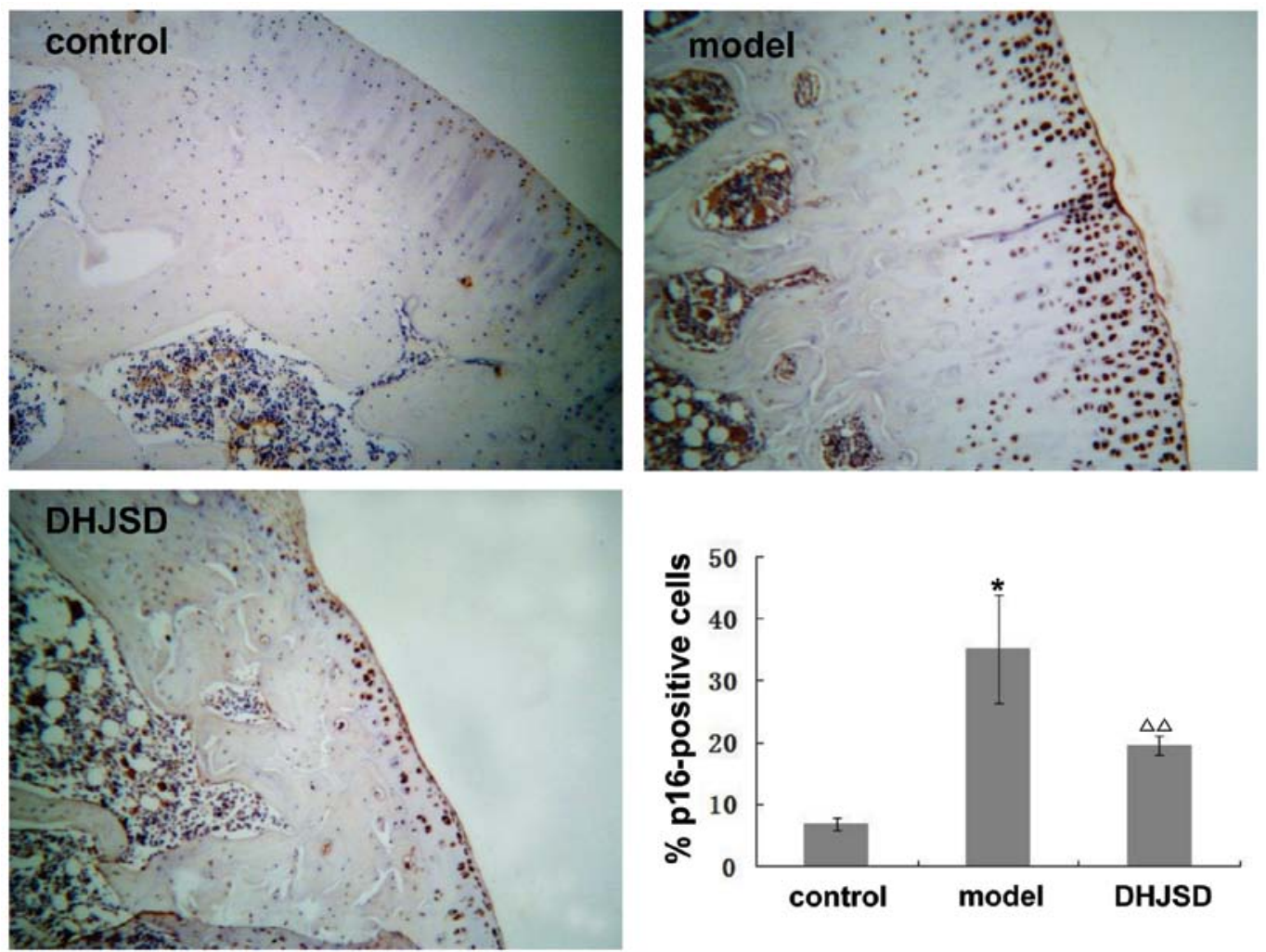

Figure 8. Effect of Duhuo Jisheng Decoction (DHJSD) on the protein expression of p16. Following treatment with or without DHJSD for 8 consecutive weeks, the rats were sacrificed and tibial plateau cartilage (inside) specimens from each group were processed for immunohistochemical staining of p16. The images were acquired at a magnification of $x 100$. Results from the quantification of immunohistochemical assay are represented as the percentage of positively-stained cells. Data shown are the means \pm SD (error bars) from 9 individual rats in each group. ${ }^{*} \mathrm{p}<0.01$, statistically significant versus control group. ${ }^{\triangle} \mathrm{p}<0.05$, statistically significant versus model group.

cytes by upregulating the expression of cyclin D1, CDK4, CDK6 and $\mathrm{Rb}$ and downregulating the expression of p16; this may be one of the mechanisms through which DHJSD exerts its therapeutic effects.

Chondrocytes are the only cell type present in mature cartilage; they are responsible for extracellular signals and regulate the maintenance of cartilage homeostasis. Therefore, the functional changes of chondrocytes play an important role by contributing to the degradation of articular cartilage and thus to the pathogenesis of OA $(27,28)$. Several studies have reported that osteoarthritic chondrocytes have a very low proliferative activity; thus, promoting chondrocyte proliferation may be an efficient treatment strategy to cure or delay the progression of OA $(29,30)$. Therefore, in the present study, we focused on chondrocyte proliferation and the anti-degenerative effects of DHJSD on cartilage. Our microstructural observation of cartilage tissue demonstrated that DHJSD is effective in the treatment of OA. Using TEM, we further clarified that the mechanisms behind the therapeutic effects of DHJSD in OA involve the promotion of chondrocyte proliferation.

The cell cycle plays an important role in the division and duplication of chondrocytes and can be divided into 4 phases: G1 phase, preparation for DNA synthesis; S phase, DNA synthesis; G2 phase, preparation for mitosis; and $\mathrm{M}$ phase, mitosis (31), where cell division occurs (32). The G1/S transition is one of the 2 main checkpoints used by a cell to regulate the progression of the cell cycle and following that, chondrocytes can pass through to the $\mathrm{S}$ phase and complete cell division (33). Progression through the G1 phase of the cell cycle is tightly regulated by the successive activation of D-type cyclins and CDKs (34). Cyclin D1 forms complexes with CDK4 or CDK6, which play an important role in the G1/S transition by phosphorylating $\mathrm{Rb}$ (35). As a consequence of $\mathrm{Rb}$ phosphorylation, $\mathrm{E}_{2} \mathrm{~F}$ is released from the $\mathrm{Rb} / \mathrm{E}_{2} \mathrm{~F}$ complexes and then triggers cell progression from the $\mathrm{G} 1$ to the $\mathrm{S}$ phase (36). During the progression of the cell cycle, CDK activity can be blocked by the binding of CDKIs, such as p16, a potent inhibitor protein of CDK/cyclin complexes, which plays an important role in S phase arrest (37). In conclusion, cell cycle regulatory factors, including cyclin D1, CDK4, CDK6, Rb and p16 may regulate the G1/S transition in chondrocytes. In this study, we demonstrate that treatment with DHJSD enhances cyclin D1, CDK4, CDK6 and Rb mRNA expression and decreases p16 mRNA expression in chondrocytes, indicating that treatment with DHJSD promotes the progression of chondrocytes from the $\mathrm{G} 1$ to the $\mathrm{S}$ phase by affecting cyclin D1, CDK4, CDK6, Rb and p16 at the transcriptional level. In order to further confirm our results, we determined the effects of DHJSD on the protein expression of cyclin D1, CDK4, CDK6, $\mathrm{Rb}$ and $\mathrm{p} 16$ by immunohistochemistry. The results revealed that the protein expression of cyclin D1, CDK4, CDK6 and Rb increased and that the expression of p16 decreased following treatment with DHJSD, which is in accordance with the pattern of their mRNA expression. 
In conclusion, to our knowledge, the data presented in this study demonstrate for the first time that treatment with DHJSD promotes the progression of chondrocytes from the G1 to the $S$ phase through the upregulation of the expression of cyclin D1, CDK4, CDK6, Rb and the downregulation of p16, suggesting that DHJSD may be a potential novel therapeutic agent for the treatment of OA.

\section{Acknowledgements}

This study was supported by the Special Research Fund for Doctor Discipline in College (20123519110001).

\section{References}

1. Heinegard D, Bayliss $M$ and Lorenzo P: Biochemistry and metabolism of normal and osteoarthritic cartilage. In: Osteoarthritis. Brandt KD, Doherty M and Lohmander LS (eds). Oxford University Press, New York, NY, pp74-84, 1998.

2. Pritzker K: Pathology of osteoarthritis. In: Osteoarthritis. Brandt KD, Doherty M and Lohmander LS (eds). Oxford University Press, New York, NY, pp50-61, 1998.

3. Kim HA and Blanco FJ: Cell death and apoptosis in osteoarthritic cartilage. Curr Drug Targets 8: 333-345, 2007.

4. Yang X, Chen L, Xu X, Li C, Huang C and Deng CX TGF- $\beta /$ Smad 3 signals repress chondrocyte hypertrophic differentiation and are required for maintaining articular cartilage. J Cell Biol 153: 35-46, 2001.

5. Iannone F, De Bari C, Scioscia C, Patella V and Lapadula G: Increased $\mathrm{Bcl}-2 / \mathrm{p} 53$ ratio in human osteoarthritic cartilage: a possible role in regulation of chondrocyte metabolism. Ann Rheum Dis 64: 217-221, 2005.

6. Zhou HW, Lou SQ and Zhang K: Recovery of function in osteoarthritic chondrocytes induced by $16^{\mathrm{INK} 4 \mathrm{a}}-\mathrm{siRNA}$ in vitro. Rheumatology 43: 555-568, 2004.

7. Tetlow LC and Woolley DE: Histamine stimulates the proliferation of human articular chondrocytes in vitro and is expressed by chondrocytes in osteoarthritic cartilage. Ann Rheum Dis 62: 991-994, 2003

8. Hulleman E, Bijvelt JJ, Verkleij AJ, Verrips CT and Boonstra J: Nuclear translocation of mitogen-activated protein kinase p42MAPK during the ongoing cell cycle. J Cell Physioll 180: 325-333, 1999.

9. Ekholm SV, Zickert P, Reed SI and Zetterberg A: Accumulation of cyclin $\mathrm{E}$ is not a prerequisite for passage through the restriction point. Mol Cell Biol 21: 3256-3265, 2001.

10. Massagué J: G1 cell-cycle control and cancer. Nature 432 : 298-306, 2004.

11. Tashiro E, Tsuchiya A and Imoto M: Functions of cyclin D2 as an oncogene and regulation of cyclin D1 expression. Cancer Sci 98 : 629-635, 2007.

12. Musgrove EA, Caldon CE, Barraclough J, Stone A and Sutherland RL: Cyclin D as a therapeutic target in cancer. Nat Rev Cancer 11: 558-572, 2011.

13. Li J, Poi M and Tsai MD: Regulatory mechanisms of tumor suppressor P16(INK4A) and their relevance to cancer. Biochemistry 50: 5566-5582, 2011.

14. Witkiewicz AK, Knudsen KE, Dicker AP and Knudsen ES The meaning of p16(ink4a) expression in tumors: functional significance, clinical associations and future developments. Cell Cycle 10: 2497-2503, 2011.

15. Bensky D, Clavey S, Stöger E and Gamble A: Chinese Herbal Medicine: Materia Medica. 3rd edition. Eastland Press, Seattle, WA, 2004.

16. Ho LJ and Lai JH: Chinese herbs as immunomodulators and potential disease-modifying antirheumatic drugs in autoimmune disorders. Curr Drug Metab 5: 181-192, 2004.
17. Peng HR: Grand Dictionary of Chinese Medicinal Formula (Zhong Yi Fang Ji Da Ci Dian). Vol. 1. 1st edition. People's Health Press, Beijing, p1113, 1993.

18. Scheid V, Bensky D, Ellis A and Barolet R: Chinese Herbal Medicine: Formulas and Strategies. 2nd edition. Eastland Press, Seattle, WA, 2009.

19. Sun SM: Bei Ji Qian Jin Yao Fang. Vol. 8. 1st edition. People's Medical Publishing House, Beijing, pp166-167, 1982.

20. Zheng CS, Xu XJ, Ye HZ, Wu GW, Li XH, Huang SP and Liu XX: Computational approaches for exploring the potential synergy and polypharmacology of Duhuo Jisheng Decoction in the therapy of osteoarthritis. Mol Med Rep 7: 1812-1818, 2013.

21. The Ministry of Science and Technology of the People's Republic of China: Guidance Suggestions for the Care and Use of Laboratory Animals, 2006.

22. Murat N, Karadam B, Ozkal S, Karatosun V and Gidener S: Quantification of papain-induced rat osteoarthritis in relation to time with the Mankin score. Acta Orthop Traumatol Turc 41: 233-237, 2007.

23. Garstang SV and Stitik TP: Osteoarthritis: epidemiology, risk factors, and pathophysiology. Am J Phys Med Rehabil 85 (Suppl 11): S2-S11, 2006.

24. Chan E, Tan M, Xin J, Sudarsanam S and Johnson DE: Interactions between traditional Chinese medicines and Western therapeutics. Curr Opin Drug Discov Devel 13: 50-65, 2010.

25. Khanna D, Sethi G, Ahn KS, Pandey MK, Kunnumakkara AB, Sung B, Aggarwal A and Aggarwal BB: Natural products as a gold mine for arthritis treatment. Curr Opin Pharmacol 7: 344-351, 2007.

26. Huh JE, Lee WI, Seo BK, Baek YH, Lee JD, Choi DY and Park DS: Gastroprotective and safety effects of WIN-34B, a novel treatment for osteoarthritis, compared to NSAIDs. J Ethnopharmacol 137: 1011-1017, 2011.

27. Shortkroff S and Yates KE: Alteration of matrix glycosaminoglycans diminishes articular chondrocytes' response to a canonical Wnt signal. Osteoarthritis Cartilage 15: 147-154, 2007.

28. Chan BY, Fuller ES, Russell AK, et al: Increased chondrocyte sclerostin may protect against cartilage degradation in osteoarthritis. Osteoarthritis Cartilage 19: 874-885, 2011.

29. Huang JG, Xia C, Zheng XP, et al: $17 \beta$-Estradiol promotes cell proliferation in rat osteoarthritis model chondrocytes via PI3K/Akt pathway. Cell Mol Biol Lett 16: 564-575, 2011.

30. Kashiwagi A, Schipani E, Fein MJ, Greer PA and Shimada M: Targeted deletion of Capn 4 in cells of the chondrocyte lineage impairs chondrocyte proliferation and differentiation. Mol Cell Biol 30: 2799-2810, 2010.

31. Zhang M, Xie R, Hou W, et al: PTHrP prevents chondrocyte premature hypertrophy by inducing cyclin-D1-dependent Runx2 and Runx3 phosphorylation, ubiquitylation and proteasomal degradation. J Cell Sci 122: 1382-1389, 2009.

32. Lee HP, Chen YL, Shen HC, Lo WH and Hu YC: Baculovirus transduction of rat articular chondrocytes: roles of cell cycle. J Gene Med 9: 33-43, 2007.

33. Löwenheim H, Reichl J, Winter $\mathrm{H}$, et al: In vitro expansion of human nasoseptal chondrocytes reveals distinct expression profiles of G1 cell cycle inhibitors for replicative, quiescent, and senescent culture stages. Tissue Eng 11: 64-75, 2005.

34. Aszodi A, Hunziker EB, Brakebusch C and Fässler R: Beta1 integrins regulate chondrocyte rotation, G1 progression, and cytokinesis. Genes Dev 17: 2465-2479, 2003.

35. Singh S, Johnson J and Chellappan S: Small molecule regulators of Rb-E2F pathway as modulators of transcription. Biochim Biophys Acta 1799: 788-794, 2010.

36. Bhaduri S and Pryciak PM: Cyclin-specific docking motifs promote phosphorylation of yeast signaling proteins by $\mathrm{G} 1 / \mathrm{S}$ Cdk complexes. Curr Biol 21: 1615-1623, 2011.

37. Liu D, Liu J, Lin B, et al: Lewis y regulate cell cycle related factors in ovarian carcinoma cell RMG-I in vitro via ERK and Akt signaling pathways. Int J Mol Sci 13: 828-839, 2012. 